\title{
An Enumeration Method Applied in Intelligent Transportation System
}

\author{
Shengnan $\mathrm{Lu}^{1,2,{ }^{*}}$ Huansheng Song ${ }^{1}$ Xiaojuan $\mathrm{Xu}^{1}$ \\ 1 College of Information Engineering, Chang'an University, Xi'an 710064, China \\ 2 College of Computer, Xi'an Shiyou University, Xi'an 710065, China \\ *E-mail: lushengnan@xsyu.edu.cn
}

\begin{abstract}
Traditional approaches for some applications of Intelligent Transportation System(ITS), such as license plate tilt correction and vehicle speed estimation generally require feature extraction, however, feature extraction is a difficult task in complex traffic environment, and it is prone to be affected by the moving status of object and dynamic environment. In this paper, we tried a new analysis method without feature extraction for these two applications. The approach is based on enumeration. All the possible results of image analysis are tested by the testing function. Then, the extreme value of the testing function is selected and the corresponding result is just the concerning result. In the experiment, our method is applied to vehicle license plate tilt correction and vehicle speed estimation. The results demonstrate the proposed method has certain degree of robustness and validity.
\end{abstract}

Keywords: Enumeration, license plate tilt correction, vehicle speed estimation

\section{Introduction}

Over the past several years, the video surveillance has been a key part of Intelligent Transportation System (ITS), it provides a rich information source for human understanding. Extracting and analyzing visual information from a traffic surveillance video for video analysis has gained great interests in the worldwide [1].

Automatic license plate recognition (ALPR) and vehicle speed estimation are two important applications in ITS. Character recognitions are an essential and important step in an ALPR system. However, it is generally very sensitive to tilt. Therefore, tilt detection and correction are critical. This task is considered as the most important step in the ALPR system. Many researchers proposed different methods in tilt correction of license plate. Pan et al. [1] divided the slant plate image into $5 * 5$ non-overlapping blocks and the local orientation of each black was estimated by gradients of pixels in the block. The horizontal incline angle of license plate was detected by the local maximum of the direction angle histogram. Fang et al. [2] applied Canny edge detector to extract the edge of license plate image and then the Hough Transform was used to detect horizontal angle and the vertical angle. Wen et al. [3] used the connected component technique to detect large numerals and find the center position of each large numeral. Then the tilt angle of every two central points were computed and the average tilt angle was obtained for horizontal correction.

In another application of vehicle speed estimation system, in order to find the vehicle moving distance in the image, features, which could represent the object moving trajectories, must be extracted from the consecutive video sequences. Then the vehicle speed could be estimated based on the trajectories and camera calibration. Garibotto et al. [4] estimated vehicle speed from two images by tracking license plates, and the speed is calculated by using the traveled distance and time difference between the two images. Similarly, Rahim et al. [6] adopted the same method for vehicle speed estimating. However, in [6], they tracked the centroids of the moving blobs. In [7], Indu et al. first 
used optical flow algorithm to calculate changes in the intensity of the pixels of the images, and the centroid of the vehicle across the frames were obtained for speed estimating.

In the above two examples, feature extraction is an important step in processing. However, in complex traffic environment, feature extraction is prone to be affected by the moving status of vehicles and dynamic environment, such as rainy and snowy days, illumination variations and shadow effects, which will increase the difficulty of feature extraction and reduce the accuracy and robustness of process results. In this paper, we abandon the idea of feature extraction for these two applications in ITS but instead adopting a new analysis method without feature extraction. This avoids the above problems and improves the image analysis accuracy.

In this work, an efficient method based on enumeration is proposed. The remainder of this work is organized as follows. First, the basic principle of this new method is described in Sec.2. In Sec.3 and Sec.4, the new method is applied for two applications, vehicle license plate tilt correction and vehicle speed estimation. Finally, Experimental results are given in Sec.5, followed by conclusions in Sec.6.

\section{Enumeration Method}

The enumeration method is a practical analysis method in math, it used for enumerating the non-repeated, non-missed finite cases of the issue to be solved, so as to find the solution for the problem. The proposed method is exactly based on this idea of enumeration. The details are described as below.

When the image analysis results or concerning parameters of objects are limited(in a certain range), and they are discrete, the method based on enumeration will be feasible. Considering the tolerance of measurement error, all the possible results can be enumerated as a discrete set $\left\{r^{(i)}\right\}, i=1,2, \ldots, k$. Then, we can construct a mapping relationship $f$, which depends on $r^{(i)}$, shown as (1), $y$ is the result of the relationship. Therefore, for each $r^{(i)}$, there will be a corresponding $y$ constituting mapping relationship with it. Meanwhile, one condition need to be satisfied for the mapping relationship, that is the relationship has an extreme value, and the $\mathrm{r}$ corresponding to the extreme value is our concerning result or parameter.

$$
y=f\left(r^{(i)}\right), i=1,2, \ldots, k
$$

\section{Vehicle License Plate Tilt Correction}

In this section, the new enumeration method is used for vehicle license plate tilt correction. Assuming the maximum slant angle is $10^{\circ}$ in the license plates, then the rotation range is from $-10^{\circ}$ to $10^{\circ}$. When considering the tolerance is $\pm 1^{\circ}$, there will be 20 rotation results for the slant license plate. Then we try to find the best rotation angle in these 20 results, and rotate the license plate image with the rotation angle. The mapping relationship depends on the rotation angle is constructed as followed.

To find the horizontal slant angle, the roof edge of the original license plate image is firstly extracted, then the horizontal projection is obtained from the edge image. When the image rotates 1 degree, the projection value and rotation angle are recorded. The procedure is repeated until the image is rotated from $-10^{\circ}$ to $10^{\circ}$, as shown in Fig.1. Next, the sums of difference values between adjacent rows in every projection curve is recorded. Finally, we found the maximum of the sums of difference values, which corresponds to the rotation angle, and the image could be corrected based on the horizontal slant angle. 


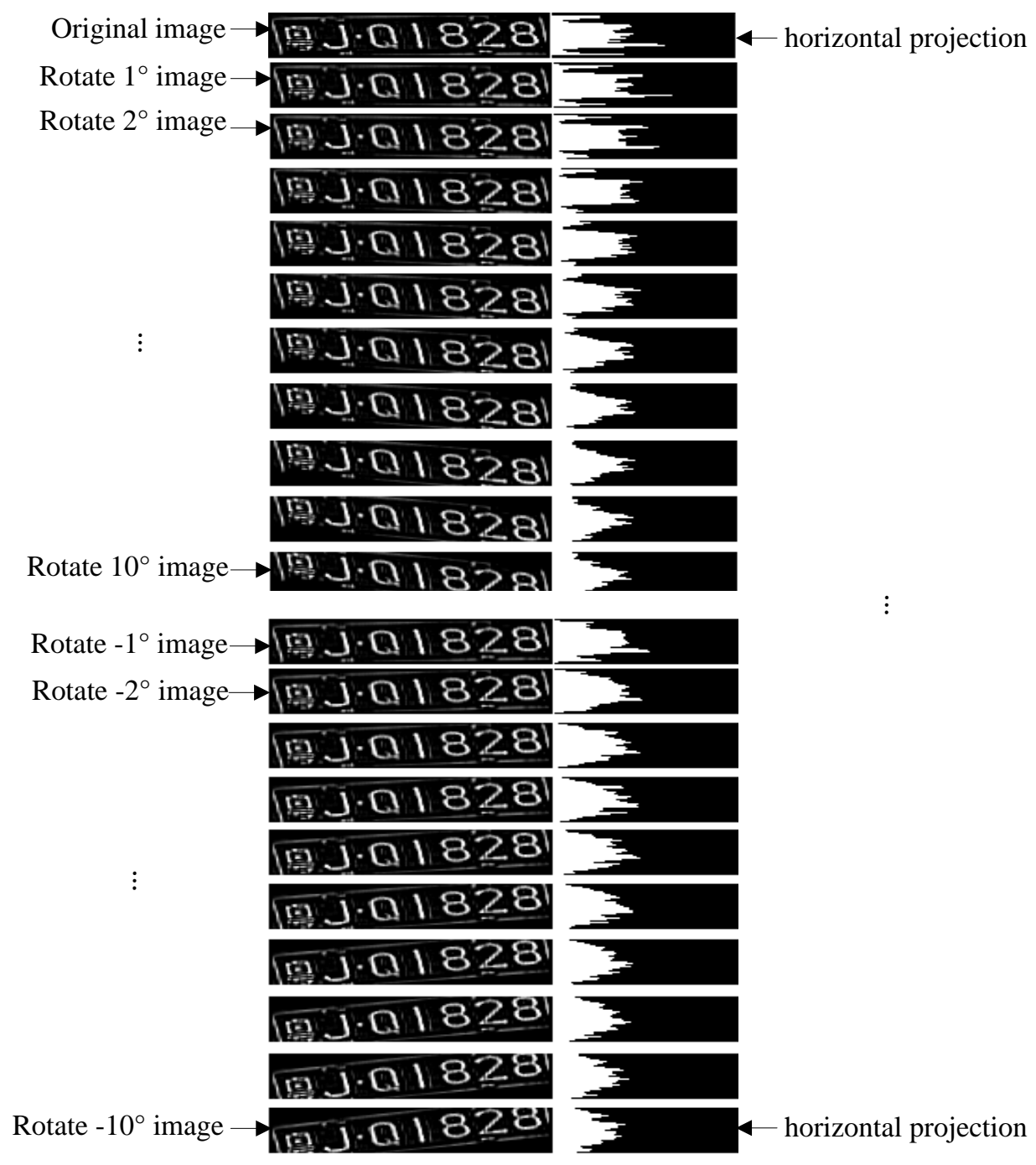

Figure 1. Image Title Correction

\section{Vehicle Speed Estimation}

In the following text, an example of vehicle speed estimation applying the new enumeration method is introduced. Similarly, vehicle speed, as a measured parameter, is usually at a range, for example $1 \sim 100 \mathrm{~km} / \mathrm{h}$ in urban. Then, we can enumerate all the possible speeds as a discrete speed set $\left\{V^{(i)}\right\}=\{1,2, \ldots, 100\}$, considering the tolerance is $1 \mathrm{~km} / \mathrm{h}$. The detailed steps for vehicle speed estimation are listed as follows.

Step 1. Establish mapping relationship between image plane and real world plane, and the relationship is stored in a mapping table.

Step 2. Select the initial tracking point manually at the rear of the vehicle, the best point is on the shadow of the bottom of the moving vehicle, which is denoted as TargetDot $(x, y)$, as shown in Figure 2.

Step 3. Set the Tracing Line and Tracing Zone. The road region has been divided along the traffic direction into 128 sub-regions by 127 detection lines, as shown in Figure 3. Tracing Line is determined by these pre-defined detection lines, and the nearest one to the TargetDot $(x, y)$ is selected, shown as the red dotted line in Figure 2. Then, a rectangle region of $m^{*} n$ pixels is generated dynamically, this region starts from point 
TargetDot $(x, y)$, and centered along the determined tracing line, here, $m=90$ and $n=17$. The region is shown as Tracing Zone in Figure 2.
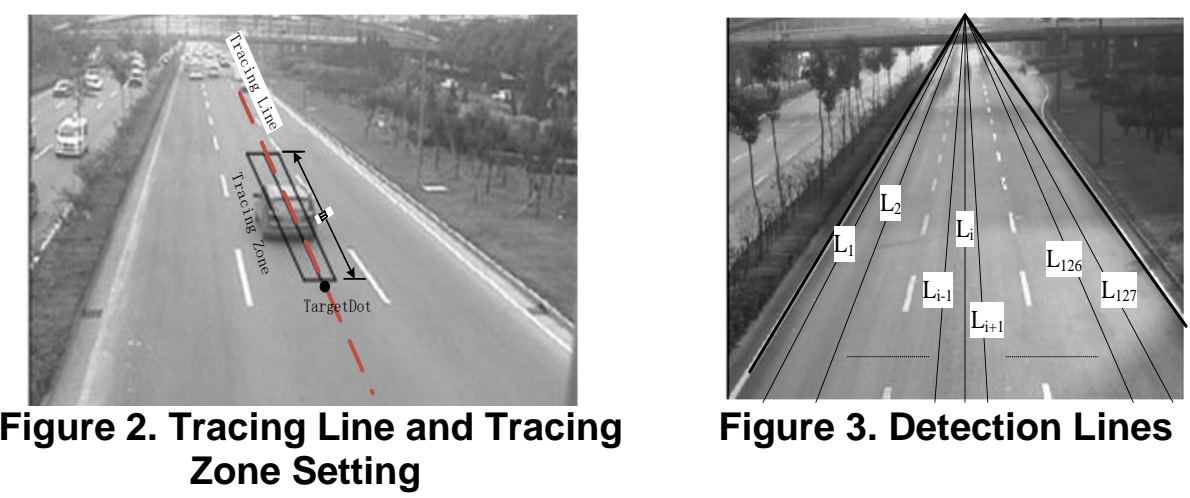

Figure 3. Detection Lines

Step 4. Extract consecutive ten video frames in Tracing Zone, as shown in Figure 4.

Step 5. Extract the target vehicle projection sequence with frame difference method. First, the difference value of the Tracing Zone is got between frames at time $k+2$ and $k$ using the frame difference method. There will be eight difference images obtained from the ten video frames in Step 4. Second, the horizontal projection histograms of eight difference images are calculated, as shown in (2), where DiffValue $[k][r]$ is the projection value of the line $r$ in the $k$ difference image and Framek $(r, i)$ is the pixel of line $r$ column $i$ in the frame $k$. We can see that the sum of difference values has not only negative values but also positive values, as shown in Figure 4. Then, the maximum of each horizontal projection histogram is extracted as feature point, shown as the red line in Figure 5. From Figure 5, it is noticed that there is a certain pattern in the projection histograms, which keeps shifting steadily with vehicle movement along moving direction. So, any point (for example the red one) can indicate the movement tendency with almost fixed relative position and value in this pattern.

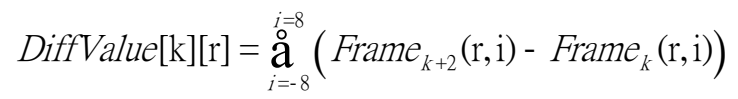

Where, $k=0,1, \ldots, 7 \quad r=0,1, \ldots, 89$

Step 6. Look up the mapping table and find the target vehicle initial position in the real world plane according to the feature point in Figure 5(a). Then, assign series of speed values $(1,2 \cdots \cdots, 99,100 \mathrm{~km} / \mathrm{h})$ to the target vehicle. For each speed, we will track 8 frames continuously and get 8 distance values. Transform these distance values to image pixels through mapping table. Finally, compare these pixels' gray (denoted as gray $_{i}$ ) values with the feature points' (denoted as $\mathrm{Gray}_{i}$ ) to find the closest one, which is considered as the real vehicle speed. The comparison principle is shown as (3). The final results are shown as the green line in Figure 5.

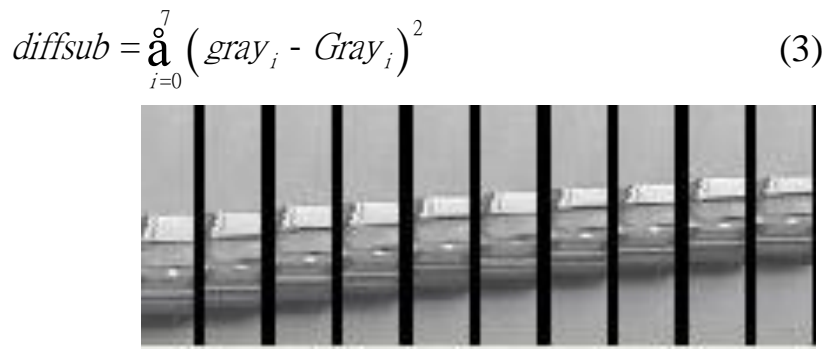

Figure 4. Image Sequence of Tracing Zone ROI 


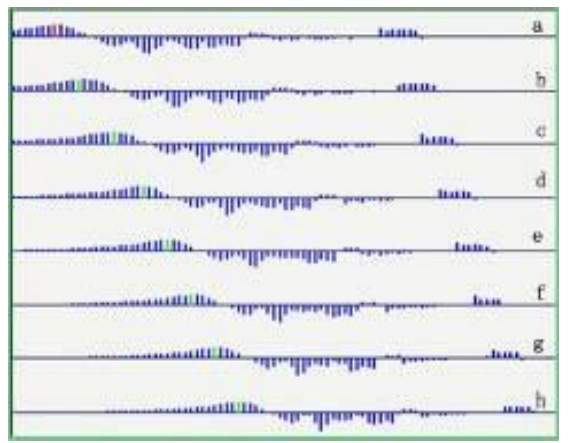

Figure 5. Projection Histograms

\section{Experiment Results}

To demonstrate the robustness and validity of the proposed method, we describe the two experiment results on real-time system. The two algorithms have been developed using Visual $\mathrm{C}++$ on a Pentium 4 3.2-GHz central processing unit (CPU) with 2-GB random access memory (RAM). The size of each image is $720 \times 288$, and the sampling frequency is $25 \mathrm{fps}$.

In the experiment of vehicle license plate tilt correction, the performance of license plate recognition system is tested with different tilt correction methods. We compared the plate recognition results of the proposed method with those of method in [3]. 2622 digital images from three sample sets with different illumination conditions are tested. The results are shown in Table I. Sample set 1 contains the actual images acquired on the road, and sample set 2 represents shadow and exposure images from the road, while sample set 3 represents natural scenes of nonmoving vehicles obtained from various parking areas. The performance of license plate recognition for each sample set and average performance of the three sample sets are presented. As shown in Table I, the performance is achieved by calculating the percentage of license plates that have been correctly identified by the machine and verified by a person supervising the test sample. Compared to sample set 1, because of shadow and exposure, the performance is lower for sample set 2. Sample set 3 contains 1568 images, which were collected under various illumination scenes and surroundings, and it still obtained 98.4\%. Besides, the performance of license plate recognition with the proposed algorithm is higher than those with the method in [3]. Overall, the license plate recognition system with the proposed algorithm achieved satisfactory results.

Table 1. A Comparison with Other Approach for License Plate Recognition

\begin{tabular}{|l|c|c|}
\hline & Proposed algorithm & Ying Wen et al. [3] \\
\hline sample set 1 & $722 / 732(98.6 \%)$ & $714 / 732(97.5 \%)$ \\
\hline sample set 2 & $314 / 322(97.5 \%)$ & $309 / 322(96 \%)$ \\
\hline sample set 3 & $1543 / 1568(98.4 \%)$ & $1532 / 1568(97.7 \%)$ \\
\hline Average performance & $98.36 \%$ & $97.44 \%$ \\
\hline
\end{tabular}

In the vehicle speed estimation experiment, we compare the testing results of the proposed algorithm with the speed measuring radar. The reference speed is detected by the speed measuring radar, the tolerance is set to $\pm 1 \mathrm{~km} / \mathrm{h}$. If the difference between the velocity detected by the proposed method and the velocity detected by radar is lower than or equal to the tolerance, the velocity can be thought to be correct. Experimental results for the estimation of vehicle speed are shown in Table II. In Table II, the accuracy ratio for vehicle velocity is calculated based on the correct detection count for velocity(DCV) 
and the total target count(TTC). A comparison with method mentioned in reference[5] for vehicle velocity detection is shown in Table II. As shown, the two methods are tested by the same speed detector with different error tolerance, and the testing condition is sunny day. Within the error tolerance, total 309 vehicles are tested and the speeds of 302 are correctly detected, the speed detection accuracy ratio can reach $97.7 \%$. The test results indicate that the accuracy ratios for vehicle velocity with the proposed method is higher than the method in reference[5]. The proposed method shows excellent accuracy performance for vehicle speed estimation.

Table 2. A Comparison with Other Approach for Vehicle Velocity Accuracy Ratio

\begin{tabular}{|l|l|l|}
\hline & Proposed algorithm & Bing-Fei Wu et al. [5] \\
\hline DCV/TTC $(*)$ & $302 / 309$ & $471 / 491$ \\
\hline Accuracy Ratio $(\%)$ & 97.7 & 95.5 \\
\hline Speed Detector & speed measuring radar & speed measuring radar \\
\hline Error Tolerance $(\mathrm{km} / \mathrm{h})$ & \pm 1 & \pm 5 \\
\hline
\end{tabular}

$\left({ }^{*}\right) \mathrm{DCV}$ is the Correct Detection Count for Velocity

$\left.{ }^{*}\right)$ TTC is the Total Target Count

\section{Conclusion}

Pervious approaches for some applications of Intelligent Transportation System(ITS), such as license plate tilt correction and vehicle speed estimation generally require feature extraction, which is a difficult task for the moving object in dynamic environment. In this paper, we have proposed a novel approach that avoids extracting features for image analysis. Our approach is based on enumeration. The idea of enumeration is ingeniously applied in the process of image analysis, and all the possible results of image analysis are tested one by one by the testing function, then, the extreme value of the testing function is selected and the corresponding result is our concerning result. The new method is applied for vehicle license plate tilt correction and vehicle speed estimation. The experimental results demonstrate the robustness and validity of the proposed method.

\section{Acknowledgement}

This work was supported in part by the Fundamental Research Funds for the Central Universities of China under Grant 2014G3242005, and the Research Fund for the Doctoral Program of Higher Education of China under Grant 20120205110001.

\section{References}

[1] R. L. Pan, X. J. Ma and L. Wang, “An Efficient Method for Skew Correction of License IPlate”, Second International Workshop on Education Technology and Computer Science, (2010).

[2] J. Fang and S. G. Dai, "A Vehicle License Plate Location and correction method Based the Characteristics of License Plate", Proceedings of the 10th world congress on Intelligent Control and Automation, (2012); Beijing, China.

[3] Y. Wen, Y. Lu and J. Q. Yan, "An Algorithm for License Plate Recognition Applied to Intelligent Transportation System", IEEE Transaction on Intelligent Transportation System, vol. 12, no. 3, (2011).

[4] G. Garibotto, P. Castello, D. Ninno and P. Pedrazzi, "Speed-vision: speed measurement by license plate reading and tracking", Proc. IEEE Intelligent Transportation Sys. Conf., (2001).

[5] B. F. Wu and J. H. Juang, "Adaptive vehicle detector approach for complex environments", IEEE transactions on intelligent transportation system, vol. 13, no. 2, (2012), pp. 817-827.

[6] H. A. Rahim, U. U. Sheikh, R. B. Ahmad, A. S. M. Zain and W. N. S. F.W. Ariffin, "Vehicle speed detection using frame differencing for smart surveillance system," 10th International Conference on Information Sciences Signal Processing and their Applications (ISSPA), (2010).

[7] S. Indu, M. Gupta and A. Bhattacharyya, "Vehicle Tracking and Speed Estimation using Optical Flow Method", International Journal of Engineering Science and Technology (IJEST), vol. 3, no. 1, (2011), pp. 429-434. 


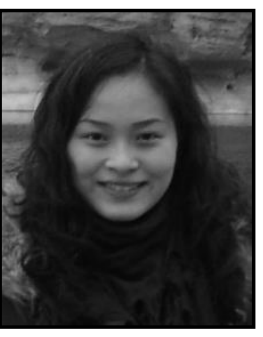

Shengnan Lu was born in JiangSu, China, in 1982. She received B.S. and M.S. degrees in computer science and technology from Chang'an University, Xi' an, China, in 2004 and 2007, respectively. She is currently working toward a Ph.D.degree in Intelligent Transportation and Information System Engineering, Chang'an University. Her research interests include image processing in intelligent transportation systems and pattern recognition. Meanwhile, she is currently a lecturer with School of Computer, Xi'an Shiyou University, Xi'an, China.

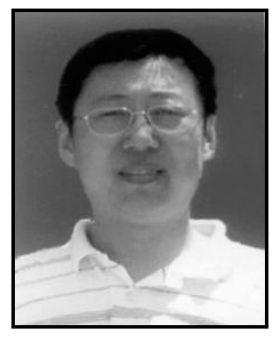

Huansheng Song was born in Inner Mongolia, China, in 1964. He received the B.S. and M.S. degrees in communication and electronic systems from Xi'an JiaoTong University, Xi'an, China, in 1985 and 1988, respectively, and the Ph.D. degree in information and communication engineering from Xi'an JiaoTong University, in 1996.

Since 2004, he has worked in the Information Engineering Institute of Chang'an University, where he became a Professor in 2006. In 2012, He was nominated as the Dean of the Information Engineering Institute. He has involved in research on intelligent transportation systems for many years and leads a research team to develop the vehicle license plate reader and traffic event detect system based on video, which has brought about complete industrialization. His current research interests include image processing and recognition and intelligent $t$ transportation systems.

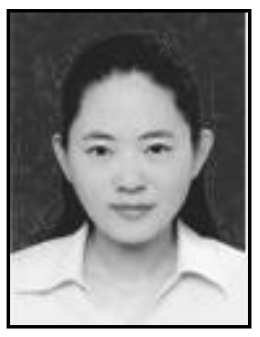

Xiaojuan Xu was born in Shandong, China, in 1988. She received the B.S. degree in Electronic Information Engineering from Chang'an University, Xi'an, China, in 2012. She is now studying for the M.S. degree in the department of Information and Communication Engineering, Chang'an University. Her research fields include computer vision, image processing and intelligent transportation system. 
International Journal of Smart Home

Vol. 9, No. 2 (2015) 